\title{
The pervasive nature of unconscious social information processing in executive control
}

\section{Ranjani Prabhakaran* and Jeremy R. Gray}

Department of Psychology, Yale University, New Haven, CT, USA

\section{Edited by:}

Nicola De Pisapia, University of

Trento, Italy

Reviewed by:

Luiz Pessoa, Brown University, USA

Pamela K. Smith, University of

California, San Diego, USA

\section{*Correspondence:}

Ranjani Prabhakaran, Department of Psychology, Yale University,

Box 208205, New Haven,

CT 06520-8205, USA.

e-mail: ranjani.prabhakaran@

yale.edu
Humans not only have impressive executive abilities, but we are also fundamentally social creatures. In the cognitive neuroscience literature, it has long been assumed that executive control mechanisms, which play a critical role in guiding goal-directed behavior, operate on consciously processed information. Although more recent evidence suggests that unconsciously processed information can also influence executive control, most of this literature has focused on visual masked priming paradigms. However, the social psychological literature has demonstrated that unconscious influences are pervasive, and social information can unintentionally influence a wide variety of behaviors, including some that are likely to require executive abilities. For example, social information can unconsciously influence attention processes, such that simply instructing participants to describe a previous situation in which they had power over someone or someone else had power over them has been shown to unconsciously influence their attentional focus abilities, a key aspect of executive control. In the current review, we consider behavioral and neural findings from a variety of paradigms, including priming of goals and social hierarchical roles, as well as interpersonal interactions, in order to highlight the pervasive nature of social influences on executive control. These findings suggest that social information can play a critical role in executive control, and that this influence often occurs in an unconscious fashion. We conclude by suggesting further avenues of research for investigation of the interplay between social factors and executive control.

Keywords: executive control, self-regulation, prefrontal cortex, visual masked priming paradigm, social priming, social power, behavioral mimicry, impression management

\section{INTRODUCTION}

Our everyday lives require a tremendous amount of deliberate behavioral regulation, including planning actions, inhibiting response tendencies, and updating goals in light of new information. These processes, known collectively as executive control, are thought to rely on the prefrontal cortex (PFC) (see Miller and Cohen, 2001 for a review) and to typically operate on consciously processed information. The definition of "consciousness" continues to serve as the topic of debate. Nonetheless, many theories of executive control view consciously processed information as that information within participants' awareness that can trigger intentional and deliberate behavioral regulation (see Jack and Shallice, 2001; Hommel, 2007). Although several studies have shown that unconsciously processed information, or information processed outside of participants' awareness, can influence a wide variety of behaviors, including semantic (e.g., Markus and Spitzer, 2000) and emotional (e.g., Whalen et al., 1998) processing, many view executive control as a higher-order function that is triggered only under conditions involving conscious awareness of conflict (Atkinson and Shiffrin, 1968; Norman and Shallice, 1986; Umilta, 1988; Dehaene and Naccache, 2001; Jack and Shallice, 2001). Additionally, the PFC has been suggested to serve as a neural correlate of consciousness, further underscoring the tight link in the literature between this brain region, executive control, and consciousness (Dehaene and Naccache, 2001; Rees et al., 2002;
Crick and Koch, 2003; Eimer and Schlaghecken, 2003). Indeed, some have viewed the very idea of unconscious executive control as an unlikely phenomenon (e.g., Dehaene and Naccache, 2001; Jack and Shallice, 2001). However, more recent evidence suggests that not only is unconscious executive control a plausible concept, but it is also a commonly occurring phenomenon in our everyday lives (Hommel, 2007; Suhler and Churchland, 2009).

Similar to the debate surrounding the definition of "consciousness," there is much controversy regarding the meaning of the term "unconscious" (see Pessoa, 2005; Bargh and Morsella, 2008). Whereas the cognitive neuroscience literature has largely focused on unconscious information processing in terms of stimuli outside of awareness (i.e., subliminal stimuli), the social psychology literature has emphasized mental processes that function outside of awareness (Bargh and Morsella, 2008; see also Nisbett and Wilson, 1977). As noted by Bargh and Morsella (2008), subliminal stimuli are weak and unnatural, thus making it difficult to assess the true scope of unconscious processing using these types of stimuli. In contrast, the social psychology literature has studied unconscious processing through investigation of how stimuli within awareness (i.e., supraliminal stimuli) can result in unconscious influences on behavior through unintentional activation of mental processes that operate outside of awareness. The results from these studies suggest that unconscious processes have a surprisingly powerful influence on our behavior (Bargh and Morsella, 2008). 
In the current review, we view unconscious executive control through the social psychological lens in order to highlight how social information can lead to unintentional triggering and engagement of executive control. First, we briefly review evidence from studies in the cognitive neuroscience literature that have used visual masked priming paradigms featuring subliminally presented visual stimuli to investigate unconscious visual information processing in executive control. We then highlight unconscious influences on executive control in the social domain, revealed via paradigms involving social priming and social interactions. It is important to note that there are both masked (i.e., subliminal) and unmasked (i.e., supraliminal) types of cognitive (e.g., visual) and social priming paradigms. For the purposes of the current review, we have focused on masked cognitive (visual) priming paradigms and unmasked social priming paradigms, as these methods have yielded several interesting findings regarding unconscious influences on executive control. Although several social psychology studies have demonstrated ways in which social factors can unconsciously have an impact on executive control, these findings have not received as much attention in the cognitive neuroscience literature. We review these findings with the aim of demonstrating the pervasive nature of unconscious social influences on executive control, as well as suggesting the need for further exploration of these influences at both behavioral and neural levels.

\section{VISUAL MASKED PRIMING PARADIGM}

Several recent studies used a visual masked priming paradigm to show that unconsciously processed visual information can trigger executive control, reflected through behavioral interference effects as well as activation of PFC. This paradigm involves presentation of a prime (e.g., a small shape) followed by a stimulus (e.g., a large hollow shape) for which participants are asked to make a response of some sort (e.g., press a button). One method of masking the prime, known as metacontrast masking, involves using a prime that has overlapping contours with the stimulus and results in reduced visibility of the prime (see Lau and Passingham, 2007; van Gaal et al., 2008, 2010; for examples of this approach). The duration of the interval between the onsets of the prime and stimulus can be altered such that a long interval increases the visibility of the prime, whereas a short interval renders the prime consciously imperceptible to the participant. Typically, the prime is either congruent or incongruent with the consciously perceptible stimulus in order to assess whether the prime can induce conflict, even though it is below the level of conscious awareness. In order to ensure that participants cannot consciously detect the primes, participants typically perform a separate discrimination task, in which they are asked to report the identity of the masked prime, after the main experimental procedure.

Interestingly, studies employing visual masked priming paradigms have shown that unconsciously perceived information can trigger executive control in a variety of different ways. For example, Lau and Passingham (2007) had participants perform either a phonological or semantic task for a presented word depending on the identity of an instruction figure shown prior to the word. When presented with a square, participants had to make a phonological judgment on the word, and when presented with a diamond, participants had to make a semantic judgment. A masked prime was presented prior to the instruction figure that was either congruent or incongruent in identity to the instruction figure. Lau and Passingham (2007) found that incongruent masked visual primes led to establishment of the incorrect task-set, reflected by impaired behavioral performance as well as activation in neural regions associated with the incorrect task and in dorsolateral prefrontal cortex (DLPFC). These results suggest that unconsciously perceived information can influence executive control-related PFC activity, thereby suggesting that consciousness is not a prerequisite for the activation of this neural region. Furthermore, as Lau and Passingham (2007) note, the establishment of task-sets and goals is a critical component of executive control (see also Miller and Cohen, 2001), which indicates that unconsciously perceived information can have a significant impact on higher-order behavior.

In addition to establishment of task-sets, studies employing visual masked priming paradigms have demonstrated that unconsciously perceived visual information can trigger inhibitory control mechanisms and related PFC activity (van Gaal et al., 2008, 2010). For example, van Gaal et al. (2010) used a visual masked priming version of a Go/No-Go task, in which participants were instructed to press a button in response to a Go stimulus (a white annulus), but to inhibit that response if a No-Go prime (a white square) appeared before the Go stimulus. The authors found that consciously imperceptible masked No-Go primes were able to increase reaction times, suggesting triggering of inhibitory control mechanisms that resulted in a slow-down in responding, compared to masked Go primes (a white diamond). Furthermore, masked No-Go trials were associated with activity in brain regions associated with inhibitory control, namely inferior frontal cortex and the pre-supplementary motor area. Thus, unconsciously perceived information appears to be able to trigger different types of executive control mechanisms and their PFC neural substrate (see also Verbruggen and Logan, 2009; Wokke et al., 2011).

\section{SOCIAL INFLUENCES ON EXECUTIVE CONTROL}

Although the studies discussed above provide compelling evidence for unconscious triggering of executive control, they have almost exclusively used visual masked priming paradigms to demonstrate these effects. In order to obtain a more comprehensive view of the relationship between unconscious processing and executive control, it is important to investigate other domains that can inform our understanding of this topic. The social psychological literature has reported unconscious influences of the social environment on a myriad of complex behaviors, including traitconsistent behaviors (e.g., walking more slowly after presentation of words related to the elderly stereotype; Bargh et al., 1996), goalpursuit (e.g., Aarts et al., 2004), and helping behavior (e.g., Garcia et al., 2002; see Ferguson and Bargh, 2004 for a review). However, the question remains as to whether social information can trigger executive control in an unconscious fashion. In the current review, we contend that not only is this a possible phenomenon, but that unconscious social influences on executive control occur in a pervasive manner in our everyday lives. 
Before we turn to the evidence supporting this conclusion, it is useful to consider two questions. First, why might social information trigger executive control? And second, why might this influence occur in an unconscious fashion? Turning to the first question, the frontal lobes of the primate brain have been suggested to support two impressive domains of human cognition: executive control as well as social cognition. The frontal lobes are thought to serve as a key neural substrate for social cognition due to the need for behavioral regulation in a manner that is sensitive to the current social context (see Adolphs, 2001, 2010 for reviews). Furthermore, it has been suggested that the evolution of the primate brain, particularly the expansion of the frontal lobes, occurred in response to the social complexity of our environments (Humphrey, 1976; Dunbar, 2003; Dunbar and Shultz, 2007). In light of the tight link between executive control and social cognition, it is perhaps not too surprising that social information can serve as a trigger of executive control. However, can this influence occur without our awareness? Humans' conscious executive control resources, although impressive in the ability to support behavior in both a flexible and goal-directed fashion, are thought to be limited in nature. Thus, the ability for social information to trigger executive control without our awareness may allow us to automatically and efficiently suppress socially inappropriate response tendencies through practice over time, thereby greasing the cogs of social interaction (Suhler and Churchland, 2009; van Gaal et al., 2010).

Given the ubiquitous nature of social influences in our environment, there are several ways in which social information can trigger executive control without our awareness. In the current review, we consider behavioral and neural evidence from paradigms involving social priming of task-sets and hierarchical roles, as well as interpersonal interactions, and their unconscious influences on executive control. We focus on evidence from two types of techniques (see Bargh and Chartrand, 2000; Smith and Galinsky, 2010 for reviews of these methods). First, we focus on priming techniques involving the unconscious activation of participants' social knowledge and subsequent carry-over effects to executive control tasks. Next, we focus on the use of social interactions as a means of activating social knowledge and unconscious carry-over effects affecting executive control in new situations.

At which levels of processing do these unconscious social influences operate? In some cases discussed in this review, social knowledge is activated unconsciously and participants also remain unaware that this information influences executive control. In other cases, processing of social information and triggering of executive control in a social situation may be conscious, but participants are not aware that this experience has an impact on executive control in a seemingly unrelated situation. Thus, although the level at which unconscious influences operate can vary across studies, what the reviewed studies have in common is that they involve unconscious carry-over effects of social information processing on executive control.

\section{SOCIAL PRIMING}

The social priming paradigm serves as a commonly used technique in the social psychology literature and involves the use of a manipulation that is designed to prime, or activates, social knowledge (e.g., a trait). The priming task can take one of many different forms, such as filling out a questionnaire in which participants are asked to provide their thoughts regarding a particular social concept, solving a scrambled sentences task where certain keywords are embedded within the sentence, or completing a lexical decision task where keywords serve as a subset of the word stimuli (see Bargh and Chartrand, 2000). Participants are then asked to complete an ostensibly unrelated task, which in actuality, serves as the dependent measure. The social priming technique assesses the impact of implicit activation of social knowledge on judgments and behaviors to the extent that participants are unaware of the influence of the priming task on the main dependent measure (Bargh, 1992; Bargh and Chartrand, 2000). The level of awareness can vary depending on the type of priming manipulation. For example, in the case of social conceptual priming, participants complete a task (e.g., scrambled sentences task) involving words (e.g., "lonely," "forgetful," "wrinkle") related to a particular social concept (e.g., the elderly stereotype; see Bargh et al., 1996). In this type of priming task, participants are aware of the words and sentences. However, they are typically unaware of the activation of social knowledge concepts as well as subsequent effects on a seemingly unrelated task. In the case of social mindset priming, participants complete a task (e.g., filling out a questionnaire asking them to reflect on a time when they had power over someone else) that explicitly asks them to think about a social concept (e.g., social power; Galinsky et al., 2003). In this type of priming task, participants are aware of the activation of social knowledge; however, they tend to remain unaware that this activation persists to influence performance on a seemingly unrelated task (see Bargh and Chartrand, 2000; Smith and Galinsky, 2010 for reviews).

Social priming techniques share much in common with visual masked priming paradigms in that both types of methods aim to uncover unconscious influences on cognitive processes. However, there are also important differences which should be noted. In the case of visual masked priming paradigms, participants are both unaware of having perceived the masked prime as well as its subsequent effects on performance. In contrast, conceptual and mindset forms of social priming often, although not necessarily, involve conscious perception of the prime; however, the influence of this knowledge on subsequent performance tends to operate in an unconscious fashion. In order to ensure the success of the experimenters' cover story that the social priming and dependent measure tasks were unrelated, participants are extensively debriefed to assess their level of awareness at the conclusion of the experiment. Although some have criticized this method of assessing unconscious processing of social information (Orne, 1962; see Ferguson and Bargh, 2004 for a review), the social priming technique has been widely accepted as a means of assessing the unconscious influences of social information on behavior.

\section{SOCIAL PRIMING OF TASK-SETS AND GOALS}

Social priming techniques have been used to demonstrate how the activation of social knowledge can unconsciously affect executive control. In a recent study, Goldfarb et al. (2011) had participants first perform two blocks of the Stroop task (Stroop, 1935), a classic measure of executive control that assesses participants' abilities 
to resolve conflict between competing incompatible responses. In this task, participants must name the ink color of a presented stimulus whose meaning is either congruent (e.g., "red" presented in red ink), incongruent ("red" presented in blue ink), or neutral (e.g., "xxxx" presented in red ink), with respect to the ink color. Participants then completed an ostensibly unrelated questionnaire, which served as the social priming manipulation. In this questionnaire, participants were asked to imagine a typical day for a severely dyslexic individual as a means of priming a "do not read" task-set. Following the priming manipulation, participants performed two additional blocks of the Stroop task. Interestingly, participants failed to show a significant Stroop effect (i.e., difference in reaction times for incongruent and congruent trials) only for the block immediately following the dyslexia priming manipulation. In a control social priming condition, participants were asked to imagine a typical day for an individual with severe mathematical difficulty, and no reduction in the Stroop effect was found for this condition. Furthermore, debriefing indicated that participants were not aware of the relationship between the social priming manipulation and the Stroop task. This study suggests that the dyslexia social priming manipulation temporarily activated a task-set and subsequent behavior in line with this task-set in the absence of participants' awareness. Thus, this study serves as a social analog to Lau and Passingham (2007) in demonstrating that social priming, similar to the visual masked priming paradigm, can establish a task-set in an unconscious fashion.

These results are in line with several previous studies demonstrating that similar to task-sets, goals can be activated in an automatic fashion, such that perceiving another individual pursuing a particular goal can lead to activation of the same goal within the perceiver (e.g., Aarts et al., 2004). This phenomenon of "goal contagion" suggests that the social environment can exert a powerful influence on our behavior, in ways that are often beyond our awareness. Goals often involve self-regulation of one's own thoughts, actions, and emotions. For example, self-regulatory abilities allow us to resist the temptation of a piece of cake when one's goal involves dieting, and enable us to study for an exam rather than attend a party when one's goal involves doing well in a course (Ackerman et al., 2009). Self-regulation is thought to rely on executive control resources, such as inhibitory control (see Baumeister, 1998; Barkley, 2001).

The examples of self-regulation provided above seem to involve difficult and deliberate choices that are conscious in nature. How might social information trigger self-regulation in an unconscious fashion? Although the bulk of research on self-regulation has focused on intra-personal self-regulatory processes, more recent studies suggest that inter-personal social factors can influence the success of self-regulation, and that this influence can occur in an unconscious fashion (see Fitzsimons and Finkel, 2010 for a review).

For example, recent work has demonstrated that social priming manipulations can influence self-regulation. Ackerman et al. (2009) had participants read either a story about a hungry waiter/waitress who had to exercise self-control by not eating the delicious food being served at the restaurant where he/she worked, or a story about a waiter/waitress who was not hungry and did not have to exercise self-control. Some participants were instructed to adopt the perspective of the waiter/waitress, whereas others were simply instructed to read the story. Participants then performed seemingly unrelated tasks requiring self-regulation in different domains, including indicating their willingness to spend money on luxury goods as well as persistence in a lexical generation task. Interestingly, the authors found that participants who adopted the perspective of the hungry waiter/waitress demonstrated less self-control (e.g., less words generated in the lexical generation task), suggesting depletion of self-regulatory resources, compared to participants who adopted the perspective of the waiter/waitress who did not have to exercise selfcontrol. In contrast, participants who merely read about the hungry waiter/waitress exercised greater self-control (e.g., more words generated in the lexical generation task), indicating a goalcontagion effect, compared to participants who read the story that did not involve self-control. These results suggest that the self-regulation of others can unconsciously influence our own self-regulatory abilities, and that the nature of this impact can depend on the extent to which we adopt the perspective of others. In sum, social information can unconsciously trigger executive control in the form of establishing task-sets and goals, as well as guiding behavior accordingly.

\section{PRIMING OF SOCIAL HIERARCHIES AND POWER DYNAMICS}

In addition to task-sets and goals, social priming methods have also been used to investigate the unconscious influence of social roles on behavior. In our everyday lives, we tend to serve a variety of social roles (e.g., teacher, student, parent, child, boss, employee, etc.) depending on our relationships with other people in our environments (Fiske, 1992). How do these roles guide our behavior? And more specifically, can these roles trigger executive control in the absence of our awareness? Below, we focus on social hierarchical roles and their unconscious influence on executive control.

Social hierarchies play an important role in our everyday lives and have an impact on how we interact with other people (Cummins, 2000). Those who have higher positions in social hierarchies tend to wield more power, in that they exert greater control over the behaviors of other people. These power dynamics tend to influence where we direct our social attention, such that individuals with less power tend to direct their attention to those who control their fates (Fiske, 1993; cf. Overbeck and Park, 2006). However, social power roles also have broader consequences on the processing of information and guidance of behaviors that are not explicitly social in nature. Indeed, power can be conceived of as a psychological construct that upon activation, results in broad downstream effects influencing how we think, feel, and behave across a variety of social and non-social contexts (Galinsky et al., 2003; Keltner et al., 2003; Smith and Galinsky, 2010). The psychological construct of power can be activated in either a conscious or unconscious fashion with similar effects on behavior (Galinsky et al., 2003). Smith and Galinsky (2010) noted that not only can power influence behavior unconsciously, but these unconscious influences also have far-reaching consequences in our daily lives. For example, individuals primed with high power roles tend to engage in more abstract thinking on both conceptual and perceptual tasks (Smith and Trope, 2006) and are also more likely 
to engage in action across a broad variety of contexts, including those unrelated to the possession of power (Galinsky et al., 2003).

A few recent studies have shown that social power roles can have an impact on executive control in the absence of participants' awareness. Guinote (2007) investigated the impact of social power roles on attentional control across a series of experiments. After completing a manipulation designed to prime either high-power or low-power social roles, such as describing a previous incident in which participants had power over others (high power) or others had power over them (low power), participants completed seemingly unrelated tasks targeting the ability to focus on taskrelevant information in the presence of distracting task-irrelevant information. For example, in one of these tasks, participants were asked to indicate the orientation (i.e., upright or inverted) of presented objects by pressing either a left or right key on a keyboard. The images of objects also contained salient task-irrelevant information in the form of a handle on either the right or left side of the object. On compatible trials, there was a match between the correct button to be pressed and the side of the object on which the handle appeared, whereas incompatible trials featured a mismatch between these dimensions. As more powerful individuals depend less upon situational constraints and have more control over their environment, Guinote (2007) hypothesized that these people may be better able to allocate their attention to taskrelevant information and ignore task-irrelevant information. In contrast, powerless individuals are more dependent upon externally defined constraints. Thus, they may need to simultaneously process multiple cues due to uncertainty regarding which cues are task-relevant or task-irrelevant. In support of these hypotheses, Guinote (2007) found that low power-primed individuals' reaction times were influenced by the compatibility of the handle position with the hand used to make the response, indicating that task-irrelevant information influenced their performance. In contrast, high power-primed individuals' reaction times did not differ between compatible and incompatible trials. These results suggest that high power-primed participants were better able to allocate attention to goal-relevant information compared to low power-primed participants.

Additional work has further explored the impact of social power roles on executive control by focusing on the effects of powerlessness on specific executive control mechanisms, including updating, inhibiting, and planning (Smith et al., 2008). Smith et al. (2008) primed social power roles through a variety of means, including assignment of participants to either superior or subordinate roles for a purported future interaction that never took place, a sentence-unscrambling task featuring words associated with either low or high power, and a writing task in which participants had to describe a situation in which they felt that they had high or low power. Across separate experiments, participants then completed executive control tasks targeting updating (n-back task), inhibition (Stroop task), or planning (Tower of Hanoi task). Smith et al. (2008) consistently found that low power-primed participants demonstrated poorer performance (i.e., higher error rates in the n-back task, higher error rates for incongruent trials in the Stroop task, greater number of moves for conflict trials in Tower of Hanoi task) compared to high power-primed participants for each specific executive control mechanism that was targeted.

In order to further target the source of executive impairments associated with low power, Smith et al. (2008) conducted an additional experiment in which participants performed one of two variants of the Stroop task. In one of these variants, participants were presented with a high proportion of congruent trials, where goal maintenance demands are high due to the ability to successfully perform the task by reading the word, thereby neglecting the goal to focus on the ink color, on the majority of trials. In the other variant, participants were presented with a high proportion of incongruent trials, thereby keeping goal maintenance demands low from trial to trial, but increasing inhibition demands. The authors found a difference in performance between high and low power-primed participants only for the Stroop task variant with a high proportion of congruent trials, with low power-primed participants demonstrating higher error rates on incongruent trials compared to high power-primed participants. Thus, the authors contend that low power-primed participants' poorer performance stemmed from goal neglect, or difficulty in maintaining a goal for a given task. Although participants in this study were randomly assigned to high or low power priming conditions, the authors contend that these findings have implications for social hierarchies within organizations. As those with low power must often rely on others for instructions or directions, they may have fewer opportunities to pursue their own goals. Smith et al. (2008) speculate that low power roles may further contribute to this disparity through impairment of the very cognitive processes necessary for goal maintenance and pursuit. Similar to Guinote (2007) findings, these results suggest that social power roles unconsciously influence executive control.

\section{NEURAL CORRELATES OF SOCIAL POWER PRIMING}

In addition to the behavioral evidence discussed above, neural evidence suggests that priming of social power roles can unconsciously trigger PFC activity that may reflect engagement of executive control mechanisms. For example, Boksem et al. (2009) demonstrated that during a priming task in which participants were asked to write about a previous situation where they had either high or low power, increased EEG activity in left (relative to right) PFC was associated with the high-power versus low-power priming task. Previous work has suggested that both high-power roles (e.g., Keltner et al., 2003) and increased left (relative to right) PFC activation (e.g., Sutton and Davidson, 1997) are associated with approach motivation. Thus, the authors speculate that high-power roles may engage PFC-mediated executive control mechanisms involved in goal-directed behavior and facilitation of approach tendencies. Although these findings indicate a link between social power roles and PFC activity, the extent to which this PFC activity reflects recruitment of executive control mechanisms remains speculative in nature. However, Boksem and colleagues have explored this issue further. Recent work by Boksem et al. (2011) suggests that low power social roles may not necessarily lead to a generalized deficit in executive control, but rather, may influence the types of executive control mechanisms that participants recruit. Boksem et al. (2011) found that participants who were assigned a lower status in an interactive time 
estimation task demonstrated a stronger medial frontal negativity, an event-related potential that serves as a measure of participants' evaluation and monitoring of their performance, compared to high-status individuals. As noted by the authors, these findings suggest that lower status individuals are more likely to engage in monitoring and adjustment of their performance, which stands in contrast to the work of Guinote (2007) and Smith et al. (2008). However, Boksem et al. (2011) suggest that rather than having poorer executive control, low power individuals may rely on a different executive control system that is more adaptive to their more unpredictable environments. Specifically, the authors propose that low-status individuals may rely on a reactive control system, which operates in a transient fashion and is triggered in response to a stimulus. In contrast, high-status individuals may rely on a proactive control system, which involves the active, sustained maintenance of task-relevant information (see also Braver et al., 2008).

Evidence from neuroimaging studies suggests the sensitivity of PFC to cues associated with social power roles. Marsh et al. (2009) had participants perform a gender discrimination task for photographs of male and female actors depicting high-status (e.g., low brow position) and low-status (e.g., high brow position) cues. Marsh et al. (2009) found increased activity in right ventrolateral prefrontal cortex (VLPFC) and left DLPFC associated with implicit processing of high-status versus low-status cues. Marsh et al. (2009) suggest that this activation may reflect the role of PFC in guiding behavior in an appropriate fashion given the current social context. Additionally, neuroimaging evidence suggests the sensitivity of PFC to social power roles, even when they are irrelevant to task performance. Zink et al. (2008) created an explicit social hierarchy in the context of a game situation, in which participants simultaneously played a game with one of two other simulated players, one of whom was superior in status to the participant and the other who was inferior in status. The game was non-competitive in nature such that status was irrelevant to the outcome of the game. Interestingly, the authors found greater DLPFC activity when participants viewed the picture of a superior versus inferior-status player, and this difference in DLPFC activity was not observed in a nonsocial version of the task where participants played with non-human computer players. The authors suggest that DLPFC plays a role in the processing of social hierarchical information.

In sum, results from EEG, ERP, and fMRI studies demonstrate that social power roles can trigger activity in DLPFC, even when those roles are not task-relevant. In light of the abundant evidence suggesting the critical role of DLPFC in supporting executive control, the studies discussed above indicate the sensitivity of this executive control-related neural region to social hierarchies. Together with the behavioral results discussed above, these studies suggest that social power roles have far-reaching consequences for our behavior, and that these influences often occur outside of our awareness. It is important to note that the majority of these studies have speculated rather than explicitly tested that observed PFC activity reflects engagement of executive control mechanisms. However, given that the PFC's role in social cognition has been suggested to consist of the engagement of executive control to regulate social behaviors (see Adolphs, 2001), these speculations do not seem unwarranted. Nonetheless, further investigation is clearly necessary in order to elucidate the specific contributions of PFC to social power dynamics.

\section{SOCIAL INTERACTIONS}

The studies reviewed thus far have demonstrated how the activation of social knowledge can have important downstream consequences for our abilities to use executive control in seemingly unrelated situations. These studies have tended to employ subtle priming manipulations, such as reading about other individuals or performing a simple scrambled sentences task. Although impressive in terms of their subtlety, one could argue that these types of manipulations do not truly capture the social nature of our environments.

As social creatures, we spend a great deal of time interacting with others. Can these social interactions affect executive control? And can this influence occur in an unconscious fashion? Below, we review evidence suggesting that social interactions can trigger executive control in surprising ways, and that this influence occurs without our awareness more often than we may think. The results of several studies indicate that not only the types of social interactions, but also the types of interaction partners, can unconsciously influence executive control. In particular, we consider how subtle aspects of our social interactions, such as interpersonal coordination and behavioral mimicry, as well as the gender and race of our interaction partners can trigger executive control in the absence of our awareness.

\section{TYPES OF SOCIAL INTERACTIONS High-maintenance social interactions}

Our daily lives often involve working on tasks with other individuals. For example, we may work with others in order to design an experiment or write a paper at work, or cook a meal with friends at home. Although working with others can increase efficiency, sometimes social coordination is inefficient and taxing in nature. Recent work suggests that the nature of social interactions involving coordination can affect self-regulation, which as noted above, is thought to rely on executive control mechanisms. Due to the limited nature of self-regulatory resources, our deployment of these resources comes at a cost, such that executive control may be depleted for subsequent situations requiring the same resources (Muraven and Baumeister, 2000; Baumeister, 2002; cf. Job et al., 2010). Thus, the depletion of these resources due to an effortful social interaction can result in fewer resources available for subsequent situations, even those that are non-social in nature.

Under this hypothesis, recent work has investigated the impact of high-maintenance social interactions, which involve inefficient social coordination, on self-regulation. Finkel et al. (2006) had participants first engage in either a high- or low-maintenance interaction with a confederate in the context of a collaborative task. In high-maintenance interactions, confederates impeded social coordination (e.g., deliberately making errors when giving directions to the participant), whereas in low-maintenance interactions, confederates facilitated social coordination (e.g., making no errors when giving directions and remaining in sync with the participant). After the social interaction, participants performed ostensibly unrelated tasks designed to assess 
self-regulatory abilities, such as preference for easy versus challenging tasks, and performance on an anagram task and Graduate Record Examination questions. Across studies, the authors found that high-maintenance social interactions resulted in impaired self-regulation on subsequent unrelated tasks. Furthermore, this effect was not mediated by conscious awareness of the highmaintenance nature of the social interaction, which was assessed via participants' self-reports of subjectively experienced depletion, liking for their interaction partner, or mood. The authors suggest that this effect may stem from the depletion of selfregulatory resources during high-maintenance interactions, as individuals may need to resist the temptation to discontinue the interaction or respond in socially inappropriate ways. These results suggest that high-maintenance social interactions can deplete self-regulatory resources without our awareness, and that this impact extends to subsequent unrelated situations that also rely on self-regulatory abilities.

\section{Behavioral mimicry}

Behavioral mimicry serves as another example of an unconscious influence of social interactions on executive control. We tend to mimic the behaviors of others, such as imitation of others' physical gestures and mannerisms, and this can facilitate social interactions (Chartrand and Bargh, 1999; Chartrand and Dalton, 2009). Behavioral mimicry is thought to operate via implicit schemas activated in an automatic and context-dependent fashion (Bavelas et al., 1986; Bernieri, 1988; Chartrand and Bargh, 1999; Lakin et al., 2003). However, situations in which mimicry violates social norms disables the use of schemas, and as a result, social interactions proceed in a more effortful fashion.

Dalton et al. (2009) examined the cognitive consequences of violation of mimicry norms in a series of experiments, with a focus on the impact of behavioral mimicry on the availability of executive and self-regulatory resources (see also Finkel et al., 2006, Experiment 5). In one experiment, Dalton et al. (2009) investigated the impact of social power roles, in the context of social interactions, on executive control. As noted above, priming of social power roles can trigger executive control. Power dynamics also provide a set of social norms that tend to guide behavior in ways appropriate to one's social role. For example, those people in social roles associated with less power, such as workers, tend to mimic the behavior of those people in social roles associated with more power, such as leaders (Cheng and Chartrand, 2003). The extent to which these norms are followed can have downstream effects on executive control, and strikingly, these effects can occur in an unconscious fashion. Thus, going beyond the priming of power roles, power dynamics can unconsciously affect executive control in the context of social interactions with others.

Dalton et al. (2009) manipulated social roles by assigning some participants to be leaders for a future interaction which did not take place and was only mentioned as a means of manipulating social roles. Other participants were assigned to be workers, where a confederate played the complementary social role (e.g., a worker, if the participant had been designated as a leader). Participants and confederates then completed a joint photo description task, where the confederate either did or did not behaviorally mimic the participant. For the condition featuring behavioral mimicry, the confederate mimicked the postures, gestures, and mannerisms of the participant, including crossing arms and legs, as well as touching one's own face and hair. For the no-mimicry condition, the confederate remained behaviorally out of sync with the participant, such that there was a constant mismatch in physical behaviors between the confederate and participant throughout the joint task. The authors found that when behavioral mimicry violated social norms (i.e., the leader confederate behaviorally mimicked the worker participant, or the worker confederate did not behaviorally mimic the leader participant), participants demonstrated greater interference (i.e., larger difference in reaction times between incongruent and neutral trials) on a subsequent Stroop task. Furthermore, none of the debriefed participants indicated awareness of being mimicked during the photo description task, suggesting that the impact of behavioral mimicry on executive control occurred in an unconscious fashion. These results expand on the findings of Finkel et al. (2006) to demonstrate the powerful impact of inefficient or counter-normative social interactions on executive control, even in situations where participants are not aware of the high-maintenance nature of the social interaction.

\section{IMPRESSION MANAGEMENT AND IDENTITY OF INTERACTION PARTNERS}

Another important aspect of our everyday lives concerns how we present ourselves to others. Self-presentation (i.e., impression management) refers to the processes involved in managing the impression that one presents to others, and has important consequences for several aspects of our daily lives, including making friends, developing romantic relationships, getting a job, and acquiring other social and financial rewards or outcomes (Baumeister, 1982; Leary and Kowalski, 1990; Vohs et al., 2005). Importantly, impression management can differ depending on the social context and can also vary in terms of the level of awareness involved (see Baumeister, 1982; Schlenker and Weigold, 1992 for reviews). In familiar situations, such as talking with a good friend, we tend to rely on well-practiced behaviors that can proceed in an automatic, effortless fashion. However, in unfamiliar or novel situations, such talking to the president of the company where we work, we may have to rely on more effortful processing in order to effectively control our behaviors to be in line with the impression that we wish to convey.

Such effortful impression management has been suggested to rely on self-regulatory resources and to have important consequences for subsequent situations reliant on self-regulation (Vohs et al., 2005). In order to test this hypothesis, Vohs et al. (2005) conducted a series of experiments in which participants first engaged in effortful, counter-normative self-presentation situations, such as presenting oneself in a modest fashion to a stranger or in a self-enhancing fashion to a friend. Participants then completed a variety of seemingly unrelated measures designed to assess self-regulatory abilities, such as persistence on a set of math problems and suppression of emotional responses while watching an upsetting video. Across these experiments, the authors found that self-presentation in unfamiliar or counternormative situations resulted in less self-regulatory ability on a subsequent task. Furthermore, the authors tested whether this relationship held in 
the other direction. For example, participants first performed the Stroop task, followed by a seemingly unrelated task involving a social interaction. The authors found that taxing self-regulatory resources in this manner led to inappropriate self-presentation in a subsequent social interaction. In sum, these experiments provide evidence suggesting that impression management can affect executive control in an unconscious fashion.

\section{Impression management: opposite-sex interactions}

Several studies have elaborated on the work of Vohs et al. (2005) to show how impression management depending on the identity of interaction partners affects executive control in subsequent tasks. For example, for heterosexual individuals, interacting with a member of the opposite sex can often involve high levels of impression management concerns (Baumeister, 1982; Bruch et al., 1989). Karremans et al. (2009) recently investigated the impact of impression management associated with opposite-sex interactions on performance on executive control tasks assessing updating (n-back task) as well as task-switching and inhibition (modified Simon task). In two experiments, the authors found that heterosexual male participants who engaged in a brief interaction with an opposite-sex confederate demonstrated poorer performance on subsequent executive control tasks compared with male participants who interacted with a same-sex confederate. It is important to note that similar to the experiments conducted by Vohs et al. (2005), Karremans et al. (2009) led participants to believe that the social interaction and executive control task components of the experiment were unrelated, suggesting that the influence of the social interaction on executive control operated on an unconscious level.

Interestingly, recent work shows that even the presence of another individual within a specific socio-evaluative context can affect executive control. Dreisbach and Bottcher (2011) had female participants perform a flanker task (Eriksen and Eriksen, 1974), in which participants were asked to indicate the direction of a central left or right pointing arrow that was flanked by arrows pointing in either the same (compatible trials) or different (incompatible trials) direction. Each trial was preceded by a picture of either a landscape or an attractive woman. Concurrently, a fellow student or the romantic partner of the participant evaluated the attractiveness of either the landscapes or the pictures of women. The fellow student or romantic partner performed the evaluation task in the same room as the participant, and was given verbal instructions for the evaluation task such that the participant could hear the instructions. The authors found that participants demonstrated impaired performance on incompatible flanker trials (those trials that invoke executive control) only following pictures of attractive women, but not landscapes. Furthermore, this impairment was only observed in situations where a fellow student or the participant's romantic partner concurrently rated the attractiveness of the pictures of women, but not landscapes. The authors interpret the selectivity of these results as stemming from participants' affective reaction to concurrent evaluation of socially threatening stimuli. Thus, this study suggests the importance of the social context within which we exert executive control. As the authors note, humans typically tend to operate within a social context. Consequently, our social environments are likely to have a pervasive influence on our abilities to exert executive control, even in tasks that are seemingly unrelated to the current social context.

\section{Impression management: interracial interactions}

In addition to the sex of our interaction partners, the work of Richeson and colleagues demonstrates that the race of one's interaction partner can also trigger executive control. Richeson and Shelton (2003) found that participants with higher levels of implicit racial bias, assessed via the Implicit Association Test (IAT; Greenwald et al., 1998), demonstrated poorer executive control (i.e., greater Stroop interference) following an interracial interaction than after an interaction with someone of the same race. These results suggest that interracial interactions can have an impact on executive control in seemingly unrelated situations where race is no longer a part of the social context.

In a subsequent study, Richeson et al. (2003) investigated the neural mechanisms underlying the relationship between racial bias, interracial interactions, and executive control. Participants first took part in a behavioral testing session, in which they completed the IAT as well as the Stroop task, following either a same-race or inter-racial interaction with a confederate. At least two weeks later, participants were contacted by a different experimenter and asked to take place in an ostensibly unrelated fMRI study, in which they viewed unfamiliar black and white faces in the context of a spatial perception task. The authors found that neural activity in lateral PFC and the anterior cingulate cortex (ACC) in response to viewing pictures of unfamiliar black, but not white, faces predicted participants' Stroop interference effects following an interracial, but not a same-race, interaction. Importantly, the authors demonstrated that lateral PFC activity was a significant mediator of the relationship between racial bias scores and Stroop interference, suggesting that interracial interactions may temporarily tax executive control abilities supported by PFC, thus leading to impaired Stroop performance.

Although the results of the studies discussed above suggest that interracial interactions can impact executive control, the nature of this influence remains unclear. That is, why might interracial interactions lead to impaired executive control? In follow-up work, Richeson and Trawalter (2005) showed that this relationship appears to stem from the depletion of self-regulation. In one experiment, the authors increased the self-regulatory demand of interracial interactions, by providing participants with false negative feedback about the extent of prejudice revealed by their IAT scores, prior to having the participants engage in the interaction. This led to greater subsequent Stroop interference compared to a control condition in which participants were provided with false negative feedback about their IAT scores that was unrelated to prejudice. In a separate experiment, the authors found that reducing self-regulatory demand, accomplished by providing participants with a script for a discussion about a racially sensitive topic with a black confederate, resulted in less subsequent Stroop interference compared to a control condition in which participants were not provided with a script. Importantly, the manipulation of self-regulatory demands had an impact on Stroop performance only after interracial, but not same-race, interactions. This work suggests potential mechanisms through 
which interracial interactions may trigger and deplete executive control, namely via anxiety or concern regarding the appearance of being prejudiced (see also Vorauer et al., 2000; Blascovich et al., 2001). Although the majority of the work of Richeson and colleagues has focused on racial bias in white participants, it is important to note that they have also found a depleting impact of implicit racial bias on executive control in black participants following interracial interactions (Richeson et al., 2005). In sum, the work of Richeson and colleagues suggests that interracial interactions can serve as an unconscious trigger of executive control mechanisms, leading to a temporary depletion of these resources.

Interestingly, interracial interactions can also trigger executive control through violation of social mimicry norms (Dalton et al., 2009). As same-race interactions are typically associated with a greater extent of behavioral mimicry compared to interracial interactions, those situations that feature a violation of these mimicry norms can trigger executive control. For example, Dalton et al. (2009) found that same-race interactions featuring a lack of behavioral mimicry and inter-racial interactions featuring higher levels of behavioral mimicry than normal were associated with greater subsequent Stroop interference. These results suggest that counter-normative behavioral mimicry triggered executive control depending on the correspondence between the level of behavioral mimicry and the race of one's interaction partner.

The results discussed thus far may lead one to think that interracial interactions inevitably lead to negative consequences for executive control. Thus, these findings may serve as cause for concern. However, it is important to note that not all interracial interactions necessarily lead to depletion of executive control. In particular, the context in which these interactions occur has the potential to influence their impact on executive control (Babbitt and Sommers, 2011; see also Trawalter et al., 2009). Babbitt and Sommers (2011) manipulated the interaction context for interracial and same-race (all white) dyads to either have a social focus or a task focus. Following the interaction, participants completed the Stroop task as a measure of executive control. The results of this study indicate that the task-focus condition led to a significant decrease in the extent of executive control depletion (i.e., smaller Stroop interference effects) compared to the social-focus condition for black participants in interracial dyads. Although a similar pattern was observed for whites, this effect did not reach significance. In follow-up experiments, Babbitt and Sommers (2011) found that manipulation of interaction context led to changes in self-reported concerns. In particular, white participants reported less concern about coming across as prejudiced, and black participants reported less concern about experiencing prejudice in the task-focus versus social-focus conditions. This study provides evidence suggesting that the framing or context of interracial interactions can have important downstream consequences for executive control. In particular, these results suggest an effective means of reducing the tendency of interracial interactions to deplete executive control.

\section{SOCIAL INTERACTIONS: POSITIVE IMPACT ON EXECUTIVE CONTROL?}

Babbitt and Sommers (2011) suggest one possible method of reducing the depleting effects of interracial interactions on executive control. But do social interactions always result in the depletion of executive control resources? Using the metaphor of self-regulatory or executive control resources as a muscle (Muraven and Baumeister, 2000), are there situations in which exercising this muscle can lead to facilitation, rather than fatigue, in subsequent situations reliant on executive control? If so, what types of situations lead to boosts in executive control?

Given the reliance of perspective-taking abilities on executive control (see Decety and Jackson, 2004 for a review), recent work has investigated the hypothesis that social situations that encourage perspective-taking lead to boosts in executive control. Ybarra et al. (2011) primed participants with either cooperative or competitive social goals via description of a future social interaction in either cooperative or competitive terms. This future interaction, in fact, never took place and was used only to prime participants' tendency to engage in perspective-taking. Participants then engaged in a 10 min social interaction with a confederate, after which they completed a seemingly unrelated measure of executive control (Trail Making Test). Ybarra et al. (2011) showed that participants primed with a cooperative social goal demonstrated greater executive control performance compared to those participants in the competitive condition and a no-intervention control condition. Across two follow-up experiments, Ybarra et al. (2011) showed that competitive interactions can also lead to facilitated executive control, provided that participants are encouraged to engage in perspective-taking during these interactions. Based on these results, the authors suggest that situations that encourage perspective-taking can lead to boosts in executive control, perhaps by "warming up" this shared neural resource. However, the authors suggest that challenging social interactions (e.g., interracial interactions for those with higher racial bias) or those that result in withdrawal and a focus on the self rather than on the perspective of another, can lead to depletion of executive control resources.

\section{CONCLUSIONS AND FUTURE DIRECTIONS}

As noted in the Introduction, much of the cognitive literature on unconscious information processing in executive control has focused on evidence from visual masked priming paradigms. In this review, we have discussed evidence from several social psychology studies demonstrating that in addition to stimuli outside of awareness, unconscious information processing can also refer to mental processes that operate without awareness (see Bargh and Morsella, 2008). In particular, we have considered converging evidence from the social domain and highlighted both the pervasive and unconscious influences of social factors on behavioral and neural correlates of executive control. These social factors represent common elements of our everyday lives, including social power roles as well as interactions involving social coordination, behavioral mimicry, and interactions with individuals of the opposite sex and other races.

In addition to recognizing that social factors can unintentionally influence executive control, it is important to consider how this may occur. The studies we have discussed in this review suggest some mechanisms. Social information can unintentionally trigger the establishment of task-sets (e.g., Goldfarb et al., 2011) and pursuit of goals, including those involving self-control (e.g., Ackerman et al., 2009). These task-sets and goals, established via 
social means such as reading about another individual or trying to adopt someone's perspective, can then guide behavior in a goal-consistent fashion. Information pertaining to social hierarchies and social power roles can also unconsciously influence executive control abilities, such as active maintenance of task- or goal-relevant information (e.g., Guinote, 2007; Smith et al., 2008) and related PFC activity (e.g., Boksem et al., 2009). As noted by Miller and Cohen (2001), the establishment and maintenance of goals and subsequent biasing of behavior in line with these goals serves as the principal function of PFC.

Another way in which social information can affect executive control involves the depletion of self-regulatory resources. As suggested by Vohs et al. (2005); Finkel et al. (2006); and Dalton et al. (2009) social interactions proceeding in an inefficient or counter-normative fashion may deplete self-regulatory resources. As a result, this depletion can have an impact on subsequent tasks dependent on these same self-regulatory resources, such as executive control tasks. Work by Richeson and colleagues (e.g., Richeson et al., 2005) has also shown that interracial interactions may deplete self-regulatory resources, perhaps due to concerns about appearing prejudiced, and lead to subsequent executive control impairments. Furthermore, activity in PFC appears to mediate this relationship (Richeson et al., 2003).

The studies discussed in this review have demonstrated several ways in which social factors can influence executive control in an unconscious fashion. However, they also suggest additional questions and avenues for further research. We offer two such avenues here. First, which factors determine whether a social interaction impairs or boosts executive control? Preliminary evidence suggests that interactions involving perspective-taking can lead to facilitation of executive control (Ybarra et al., 2011). This finding suggests the potential for using perspective-taking manipulations to offset the self-regulatory depletion caused by effortful interactions, such as during a high-maintenance interaction, or when interacting with someone of a different race. Further research is necessary to determine other social factors that can boost executive control. Dalton et al. (2009) have suggested that behavioral mimicry in the context of close relationships may serve to replenish executive control resources. The extent to which perspective-taking and behavioral mimicry can boost or replenish executive control should be tested across a variety of different social contexts, as this could have important consequences for the successful utilization of executive control and self-regulation in our daily lives. Additionally, further research is needed on the neural correlates of how social interactions affect executive control. Previous neuroimaging studies have largely been unable to investigate the effects of naturalistic social interactions on neural activity due to the constraints of functional neuroimaging methods. However, recent work has introduced novel methods for implementing online, face-to-face social interactions during fMRI scanning (Redcay et al., 2010), and these methods holds great promise for future work investigating the impact of social interactions on executive control.

Second, what is the duration of the impact of social priming and social interactions on executive control? In the case of the social priming technique, Goldfarb et al. (2011) found an impact on Stroop performance only in the block immediately following the dyslexia priming manipulation. This suggests that social priming effects are of short duration and may not have as farreaching consequences as a taxing social interaction. In further support of this idea, evidence from the power priming literature suggests that priming of power constructs yields similar, but less impactful, behavioral consequences compared with the actual possession of power (see Smith and Galinsky, 2010 for a review). The majority of studies employing social interactions have tended to investigate their impact on executive control for tasks immediately following the social interaction. Thus, the duration of these effects requires further study in order to determine the extent of their influence.

Additionally, it is important to note that although social information can unconsciously trigger executive control, this does not mean that all of our behavior is beyond our conscious control (see Suhler and Churchland, 2009). Rather, it is likely that both consciously and unconsciously processed information can trigger executive control depending on the situation, and that both consciously and unconsciously triggered control work in concert to efficiently guide behavior. Further research is clearly necessary to elucidate the similarities and differences between conscious and unconscious control mechanisms. In addition, further specification of the types of factors that can influence executive control will allow for identification of ways to combat these influences, particularly when they lead to undesirable behavioral consequences (e.g., depletion of self-regulatory resources).

Consideration of the unconscious influence of social factors on executive control holds a great deal of promise for future research. Indeed, based on the findings discussed in this review, it is critical to ask not only whether social information can unconsciously influence executive control, but also how these effects occur. In his seminal article on the social function of intellect, Humphrey (1976) described studying the recovery of vision in a rhesus monkey that had been kept in a cage in the laboratory. Although the monkey's recovery was somewhat limited within the laboratory, she showed a great improvement in spatial vision when she was allowed to spend time outdoors. Humphrey thus notes that "The limits on her recovery had been imposed directly by the limited environment in which she had been living" (1976, p. 308). In a similar fashion, a great deal of experimental psychological research involves testing participants' executive control abilities in isolation within the "cage" of the laboratory. However, in our daily lives, we are nearly always engaged in a social context. In order to better understand executive control processes, we must move beyond only studying participants in the limited social environment of the laboratory, and also consider the influence of social factors present in our everyday lives. Thus, greater consideration of the interplay between social factors and executive control may allow us to gain a deeper understanding of the mechanisms underlying regulation of human behavior.

\section{ACKNOWLEDGMENTS}

This work was supported by NSF DRL 0644131. We thank Marcia Johnson and members of the Johnson lab for their helpful comments and suggestions. 


\section{REFERENCES}

Aarts, H., Gollwitzer, P. M., and Hassin, R. R. (2004). Goal contagion: perceiving is for pursuing. J. Pers. Soc. Psychol. 87, 23-37.

Ackerman, J. M., Goldstein, N. J., Shapiro, J. R., and Bargh, J. A. (2009). You wear me out: the vicarious depletion of self-control. Psychol. Sci. 20, 326-332.

Adolphs, R. (2001). The neurobiology of social cognition. Curr. Opin. Neurobiol. 11, 231-239.

Adolphs, R. (2010). Conceptual challenges and directions for social neuroscience. Neuron 65, 752-767.

Atkinson, R. C., and Shiffrin, R. M. (1968). "Human memory: a proposed system and its control Processes," in The Psychology of Learning and Motivation, Vol 2, eds K. W. Spence and J. T. Spence (New York, NY: Academic Press), 89-195.

Babbitt, L. G., and Sommers, S. R. (2011). Framing matters: contextual influences on interracial interaction outcomes. Pers. Soc. Psychol. Bull. 37, 1233-1244.

Bargh, J. A. (1992). "Being unaware of the stimulus versus unaware of its interpretation: Why subliminality per se does not matter to social psychology," in Perception Without Awareness, eds. R. Bornstein and T. Pittman (New York, NY: Guilford Press), 236-255.

Bargh, J. A., and Chartrand, T. L. (2000). "The mind in the middle: a practical guide to priming and automaticity research," in Handbook of Research Methods in Social and Personality Psychology, eds. H. T. Reis and C. M. Judd (New York, NY: Cambridge University Press), 253-285.

Bargh, J. A., Chen, M., and Burrows, L. (1996). Automaticity of social behavior: direct effects of trait construct and stereotype activation on action. J. Pers. Soc. Psychol. 71, 230-244.

Bargh, J. A., and Morsella, E. (2008). The unconscious mind. Perspect. Psychol. Sci. 3, 73-79.

Barkley, R. A. (2001). The executive functions and self-regulation: an evolutionary neuropsychological perspective. Neuropsychol. Rev. 11, $1-29$.

Baumeister, R. F. (1982). A selfpresentational view of social phenomena. Psychol. Bull. 91, 326.

Baumeister, R. F. (1998). "The self," in Handbook of Social Psychology, 4th Edn. Vol. 2, eds D. T. Gilbert, S. T. Fiske, and G. Lindzey (New York, NY: McGraw-Hill), 680-740.
Baumeister, R. F. (2002). Ego depletion and self-control failure: an energy model of the self's executive function. Self Identity 1, 129-136.

Bavelas, J. B., Black, A., Lemery, C. R., Macinnis, S., and Mullett, J. (1986). Experimental methods for studying "Elementary motor mimicry". J. Nonverbal Behav. 10, 102-119.

Bernieri, F. J. (1988). Coordinated movement and rapport in teacherstudent interactions. J. Nonverbal Behav. 12, 120-138.

Blascovich, J., Mendes, W. B., Hunter, S. B., Lickel, B., and Kowai-Bell, N. (2001). Perceiver threat in social interactions with stigmatized others. J. Pers. Soc. Psychol. 80, 253-267.

Boksem, M. A. S., Kostermans, E., Milivojevic, B., and de Cremer, D. (2011). Social status determines how we monitor and evaluate our performance. Soc. Cogn. Affect. Neurosci. 7, 304-313.

Boksem, M. A. S., Smolders, R., and de Cremer, D. (2009). Social power and approach-related neural activity. Soc. Cogn. Affect. Neurosci. doi: 10.1093/scan/nsp006. [Epub ahead of print].

Braver, T. S., Gray, J. R., and Burgess, G. C. (2008). "Explaining the many varieties of working memory variation: dual mechanisms of cognitive control," in Variation in Working Memory, eds A. Conway, C. Jarrold, M. Kane, A. Miyake, and J. Towse (New York, NY: Oxford University Press), 76-106.

Bruch, M. A., Gorsky, J. M., Collins, T. M., and Berger, P. A. (1989). Shyness and sociability reexamined: a multicomponent analysis. J. Pers. Soc. Psychol. 57, 904-915.

Chartrand, T. L., and Bargh, J. A. (1999). The chameleon effect: the perception-behavior link and social interaction. J. Pers. Soc. Psychol. 76, 893-910.

Chartrand, T. L., and Dalton, A. N. (2009). "Mimicry: its ubiquity, importance, and functionality," in Oxford Handbook of Human Action, eds. E. Morsella, J. A. Bargh, and P. M. Gollwitzer (New York, NY: Oxford University Press), 458-483.

Cheng, C. M., and Chartrand, T. L. (2003). Self-monitoring without awareness: using mimicry as a nonconscious affiliation strategy. J. Pers. Soc. Psychol. 85, 1170-1179.

Crick, F., and Koch, C. (2003). A framework for consciousness. Nat. Neurosci. 6, 119-126.

Cummins, D. D. (2000). How the social environment shaped the evolution of mind. Synthese 122, 3-28.
Dalton, A. N., Chartrand, T. L., and Finkel, E. J. (2009). The schemadriven chameleon: how mimicry affects executive and self-regulatory resources. J. Pers. Soc. Psychol. 98, 605-617.

Decety, J., and Jackson, P. L. (2004). The functional architecture of human empathy. Behav. Cogn. Neurosci. Rev. 3, 71-100.

Dehaene, S., and Naccache, L. (2001). Towards a cognitive neuroscience of consciousness: basic evidence and a workspace framework. Cognition 79, 1-37.

Dreisbach, G., and Bottcher, S. (2011) How the social-evaluative context modulates processes of cognitive control. Psychol. Res. 75, 143-151.

Dunbar, R. I. M. (2003). The social brain: mind, language, and society in evolutionary perspective. Annu. Rev. Anthropol. 32, 163-181.

Dunbar, R. I. M., and Shultz, S. (2007). Evolution in the social brain. Science 317, 1344-1347.

Eimer, M., and Schlaghecken, F. (2003). Response facilitation and inhibition in subliminal priming. Biol. Psychiatry 64, 7-26.

Eriksen, B. A., and Eriksen, C. W. (1974). Effects of noise letters upon the identification of a target letter in a nonsearch task. Percept. Psychophys. 16, 143-149.

Ferguson, M. J., and Bargh, J. A. (2004) How social perception can automatically influence behavior. Trends Cogn. Sci. 8, 33-39.

Finkel, E. J., Campbell, W. K., Brunell, A. B., Scarbeck, S. J., and Chartrand, T. L. (2006). High-maintenance interaction: inefficient social coordination impairs self-regulation. $J$ Pers. Soc. Psychol. 91, 456-475.

Fiske, A. P. (1992). The four elementary forms of sociality: framework for a unified theory of social relations. Psychol. Rev. 99, 689-723.

Fiske, S. T. (1993). Controlling other people: the impact of power on stereotyping. Am. Psychol. 48, 621-628.

Fitzsimons, G. M., and Finkel, E. J. (2010). Interpersonal influences on self-regulation. Curr. Dir. Psychol. Sci. 19, 101-105.

Galinsky, A. D., Gruenfeld, D. H., and Magee, J. C. (2003). From power to action. J. Pers. Soc. Psychol. 85, 453-466.

Garcia, S. M., Weaver, K., Moskowitz, G. B., and Darley, J. M. (2002). Crowded minds: the implicit bystander effect. J. Pers. Soc. Psychol. 83, 843-853.

Goldfarb, L., Aisenberg, D., and Henik, A. (2011). Think the thought, walk the walk - social priming reduces the Stroop effect. Cognition 118, 193-200.

Greenwald, A. G., McGhee, D. E., and Schwartz, J. L. K. (1998). Measuring individual differences in implicit cognition: the implicit association task. J. Pers. Soc. Psychol. 74, 1464-1480.

Guinote, A. (2007). Power affects basic cognition: increased attentional inhibition and flexibility. J. Exp. Soc. Psychol. 43, 685-697.

Hommel, B. (2007). Consciousness and control: not identical twins. J. Conscious. Stud. 14, 155-176.

Humphrey, N. K. (1976). "The social function of intellect," in Growing Points in Ethology, eds P. P. G. Bateson and R. A. Hinde (Cambridge, MA: Cambridge University Press), 303-317.

Jack, A. I., and Shallice, T. (2001). Introspective physicalism as an approach to the science of consciousness. Cognition 79, 161-196.

Job, V., Dweck, C. S., and Walton, G. M. (2010). Ego depletion - is it all in your head? implicit theories about willpower affect self-regulation. Psychol. Sci. 21, 1686-1693.

Karremans, J. C., Verwijmeren, T., Pronk, T. M., and Reitsma, M (2009). Interacting with women can impair men's cognitive functioning. J. Exp. Soc. Psychol. 45, 1041-1044.

Keltner, D., Gruenfeld, D. H., and Anderson, C. (2003). Power, approach, and inhibition. Psychol. Rev. 110, 265-284.

Lakin, J. L., Jefferis, V. E., Cheng, C. M., and Chartrand, T. L. (2003). The chameleon effect as social glue: evidence for the evolutionary significance of nonconscious mimicry. J. Nonverbal Behav. 27, 145-162.

Lau, H. C., and Passingham, R. E. (2007). Unconscious activation of the cognitive control system in the human prefrontal cortex. J. Neurosci. 27, 5805-5811.

Leary, M. R., and Kowalski, R. M. (1990). Impression management: a literature review and twocomponent model. Psychol. Bull. 107, 34-47.

Markus, K., and Spitzer, M. (2000). Time course of conscious and unconscious semantic brain activation. Neuroreport 11, 2401-2407.

Marsh, A. A., Blair, K. S., Jones, M. R., Soliman, N., and Blair, R. J. R. (2009). Dominance and submission: the ventrolateral prefrontal cortex and responses to status cues. J. Cogn. Neurosci. 21, 713-724. 
Miller, E. K., and Cohen, J. D. (2001). An integrative theory of prefrontal cortex function. Annu. Rev. Neurosci. 24, 167-202.

Muraven, M., and Baumeister, R. F. (2000). Self-regulation and depletion of limited resources. Does selfcontrol resemble a muscle? Psychol. Bull. 126, 247-259.

Nisbett, R. E., and Wilson, T. D. (1977). Telling more than we can know: verbal reports on mental processes. Psychol. Rev. 84, 231-259.

Norman, D. A., and Shallice, T. (1986). "Attention to action: willed and automatic control of Behavior," in Consciousness and Self-Regulation, Vol. 4, eds R. J. Davidson, G. E. Schwartz, and D. Shapiro (New York, NY: Plenum Press), 1-18.

Orne, M. T. (1962). On the social psychology of the psychological experiment: with particular reference to demand characteristics and their implications. Am. Psychol. 17, 776-783.

Overbeck, J. R., and Park, B. (2006). Powerful perceivers, powerless objects: flexibility of powerholders' social attention. Organ. Behav. Hum. Decis. Process. 99, 227-243.

Pessoa, L. (2005). To what extent are emotional visual stimuli processed without attention and awareness? Curr. Opin. Neurobiol. 15, 188-196.

Redcay, E., Dodell-Feder, D., Pearrow, M. J., Mavros, P. L., Kleiner, M., Gabrieli, J. D. E., and Saxe, R. (2010). Live face-to-face interaction during fMRI: a new tool for social cognitive neuroscience. Neuroimage 50, 1639-1647.

Rees, G., Kreiman, G., and Koch, C. (2002). Neural correlates of consciousness in humans. Nat. Rev. Neurosci. 3, 261-270.

Richeson, J. A., Baird, A. A., Gordon, H. L., Heatherton, T. F., Wyland, C. L., Trawalter, S., and Shelton, J. N. (2003). An fMRI investigation of the impact of interracial contact on executive function. Nat. Neurosci. 6 , 1323-1328.

Richeson, J. A., and Shelton, J. N. (2003). When prejudice does not pay: effects of interracial contact on executive function. Psychol. Sci. 14, 287-290.

Richeson, J. A., and Trawalter, S. (2005). Why do interracial interactions impair executive function? A resource depletion account. J. Pers. Soc. Psychol. 88, 934-947.

Richeson, J. A., Trawalter, S., and Shelton, J. N. (2005). African Americans' implicit racial attitudes and the depletion of executive function after interracial interactions. Soc. Cogn. 23, 336-352.

Schlenker, B. R., and Weigold, M. F. (1992). Interpersonal processes involving impression regulation and management. Annu. Rev. Psychol. 43, 133-168.

Smith, P. K., and Galinsky, A. D. (2010). The nonconscious nature of power: cued and consequences. Soc. Personal. Psychol. Compass 4/10, 918-938.

Smith, P. K., Jostmann, N. B., Galinsky, A. D., and van Dijk, W. W. (2008). Lacking power impairs executive functions. Psychol. Sci. 19, 441-447.

Smith, P. K., and Trope, Y. (2006). You focus on the forest when you're in charge of the trees: power priming and abstract information processing. J. Pers. Soc. Psychol. 90, 578-596.

Stroop, J. R. (1935). Studies in interference in serial verbal reactions. J. Exp. Psychol. 18, 643-661.

Suhler, C. L., and Churchland, P. S. (2009). Control: conscious and otherwise. Trends Cogn. Sci. 13, 341-347.

Sutton, S. K., and Davidson, R. J. (1997). Prefrontal brain asymmetry: a biological substrate of the behavioral approach and inhibition systems. Psychol. Sci. 8, 204-210.

Trawalter, S., Richeson, J. A., and Shelton, J. N. (2009). Predicting behavior during interracial interactions: a stress and coping approach. Pers. Soc. Psychol. Rev. 13, 243-268.

Umilta, C. (1988). "The control operations of consciousness," in Consciousness in Contemporary Science, eds A. J. Marcel and E. Bisiach (Oxford: Oxford University Press), 334-356.

van Gaal, S., Ridderinkhof, K. R., Fahrenfort, J. J., Scholte, H. S., and Lamme, V. A. F. (2008). Frontal cortex mediates unconsciously triggered inhibitory control. J. Neurosci. 28, 8053-8062.

van Gaal, S., Ridderinkhof, K. R. Scholte, H. S., and Lamme, V. A F. (2010). Unconscious activation of the prefrontal no-go network. J. Neurosci. 30, 4143-4150.

Verbruggen, F., and Logan, G. D. (2009). Automaticity of cognitive control: goal priming in responseinhibition paradigms. J. Exp. Psychol. Learn. Mem. Cogn. 35, 1381-1388.

Vohs, K. D., Baumeister, R. F., and Ciarocco, N. J. (2005). Selfregulation and self-presentation: regulatory resource depletion impairs impression management and effortful self-presentation depletes regulatory resources. J. Pers. Soc. Psychol. 88, 632-657.

Vorauer, J. D., Hunter, A. J., Main, K. J., and Roy, S. A. (2000). Metastereotype activation: evidence from indirect measures for specific evaluative concerns experienced by members of dominant groups in intergroup interaction. J. Pers. Soc. Psychol. 78, 690-707.

Whalen, P. J., Rauch, S. L., Etcoff, N L., McInerney, S. C., Lee, M. B., and Jenike, M. A. (1998). Masked presentations of emotional facial expressions modulate amygdala activity without explicit knowledge. J. Neurosci. 18, 411-418.

Wokke, M. E., van Gaal, S., Scholte, H. S., Ridderinkhof, K. R., and Lamme, V. A. F. (2011). The flexible nature of unconscious cognition. PLoS One 6:e25729. doi: 10.1371/journal.pone.0025729

Ybarra, O., Winkielman, P., Yeh, I., Burnstein, E., and Kavanagh, L. (2011). Friends (and sometimes enemies) with cognitive benefits: what types of social interactions benefit executive functioning? Soc. Psychol. Personal. Sci. 2, 253-261.

Zink, C. F., Tong, Y., Chen, Q., Bassett, D. S., Stein, J. L., and MeyerLindenberg, A. (2008). Know your place: neural processing of social hierarchy in humans. Neuron 58, 273-283.

Conflict of Interest Statement: The authors declare that the research was conducted in the absence of any commercial or financial relationships that could be construed as a potential conflict of interest.

Received: 02 February 2012; accepted: 10 April 2012; published online: 26 April 2012.

Citation: Prabhakaran $R$ and Gray JR (2012) The pervasive nature of unconscious social information processing in executive control. Front. Hum. Neurosci. 6:105. doi: 10.3389/fnhum.2012.00105 Copyright (C) 2012 Prabhakaran and Gray. This is an open-access article distributed under the terms of the Creative Commons Attribution Non Commercial License, which permits non-commercial use, distribution, and reproduction in other forums, provided the original authors and source are credited. 